\title{
e-Phaïstos
}

e-Phaïstos

Revue d'histoire des techniques / Journal of the history

of technology

VIII-1 | 2020

Écomusée : une expansion internationale

\section{Le tissu « ndop ». Un processus de fabrication entre tradition et modernité, dans l'Ouest Cameroun}

The "Ndop" Fabric. Manufacturing Process in West Cameroon : between

Tradition and Modernity

Francine Ulrich Awounang Sonkeng et Jules Kouosseu

\section{(2) OpenEdition}

Journals

Édition électronique

URL : https://journals.openedition.org/ephaistos/7739

DOI : $10.4000 /$ ephaistos.7739

ISSN : 2552-0741

Éditeur

IHMC - Institut d'histoire moderne et contemporaine (UMR 8066)

Référence électronique

Francine Ulrich Awounang Sonkeng et Jules Kouosseu, « Le tissu «ndop ». Un processus de

fabrication entre tradition et modernité, dans l'Ouest Cameroun », e-Phaïstos [En ligne], VIII-1 | 2020,

mis en ligne le 29 avril 2020, consulté le 21 septembre 2021. URL : http://journals.openedition.org/ ephaistos/7739; DOI : https://doi.org/10.4000/ephaistos.7739

Ce document a été généré automatiquement le 21 septembre 2021.

Tous droits réservés 


\title{
Le tissu « ndop ». Un processus de fabrication entre tradition et modernité, dans l'Ouest Cameroun
}

\author{
The "Ndop" Fabric. Manufacturing Process in West Cameroon : between \\ Tradition and Modernity
}

Francine Ulrich Awounang Sonkeng et Jules Kouosseu

\begin{abstract}
À l'ouest Cameroun, au même titre que la religion, l'art a toujours revêtu une importante dimension socioculturelle. À côté des masques, les statues et les pièces de mobilier sculptées dans le bois, les Bamiléké et les Bamoun sont réputés pour leur savoir-faire textile mis en évidence à travers le tissu dit ndop. En général, le textile a toujours fait partie de l'histoire ancienne des peuples africains qui, aujourd'hui, représente un patrimoine culturel important à préserver. Le ndop ou ndoup ${ }^{1}$ est un tissu généralement constitué de longues pièces de coton pouvant mesurer de 3 à $15 \mathrm{~m}$ et résultant d'un assemblage de tissus de 15 à 20 bandes de $5 \mathrm{~cm}$ de large cousues bord à bord (Harter 1986: 27). Avec la queue de cheval décorée, il fait partie des symboles traditionnels bamiléké, celui des sociétés secrètes, de la noblesse et des cérémonies rituelles. Ses nombreux usages à la fois utilitaires et symboliques en font un élément indispensable de la culture des peuples Grassfields. Cependant, sa fabrication et son usage n'ont pas échappé à la modernité et même à l'industrialisation, altérant ainsi sa fonction symbolique et culturelle. Ainsi, quel savoir-faire avait été développé par les populations pour la fabrication de ce tissu? Quelles mutations ce savoir local a-t-il connues? Quel en est l'impact sur la symbolique et l'usage de ce tissu très prisé ? Cette communication se propose d'étudier l'origine et le processus de fabrication du ndop, ses usages, l'évolution dans le mode opératoire de sa confection et son impact.
\end{abstract}




\section{Le ndop : origines et usages}

2 La tradition textile à l'Ouest Cameroun remonte à plusieurs siècles et met en relation cette région avec le Nord-Ouest, le Nigéria et surtout le Nord Cameroun. Région textile par excellence, le Nord Cameroun joue un rôle majeur dans le processus de fabrication du ndop.

\section{Origines de l'étoffe dite ndop, processus de production : entre le nord et l'ouest Cameroun.}

$3 \quad \mathrm{Au} \mathrm{XV}$ siècle, les stands de teinture de Kano, au nord du Nigéria, ont bénéficié d'une renommée internationale. À Kano comme dans de nombreux autres centres de production textile précoloniale, l'élite politique de la ville était la principale clientèle des tisserands et teinturiers. En outre, les royautés favorisaient le développement de tissus spéciaux de luxe. Par exemple, la cour du roi Njoya des Bamoun a produit des modèles particulièrement fins de raphia cousus (Ouédraogo 2011 :5).

4 La présence du tissu ndop dans les Grassfields remonte au XIXe siècle. Bien avant la fabrication du tissu ndop à l'Ouest, un autre type de tissu batik, le ndop de Wukari, était vendu dans cette région et provenait du Nigéria (Perrois, Notué 1987 :171). Ces tissus ont servi de monnaie entre les peuples des Grassfields et ceux du Nigéria, jusqu'à une période avancée du $\mathrm{XX}^{\mathrm{e}}$ siècle, où la monnaie d'étoffe fut un phénomène qui s'est étendu sur presque toute l'Afrique de l'Ouest à côté des cauris, avant d'être remplacé progressivement par la monnaie papier ${ }^{2}$. Dans la région du Bornou, elle occupa une place particulière à partir du XIVe siècle (Barreteau 1997 :236). Le circuit d'échange du ndop de Wukari était celui de la Cross-River, par le pays Widikum.

Certaines sources suggèrent que l'industrie du ndop typiquement camerounais doit son origine à l'inspiration du roi Njoya qui débuta la production de ce tissu à Foumban. Des illustrations de ce que l'on pense être du ndop avec des motifs typiquement Bamoun semblent appuyer cette thèse. Cependant, de telles étoffes n'apparaissent pas sur des photographies de la cour de Foumban avant 1914 (Lamb 1981 :32). Le ndop, considéré comme typiquement camerounais, apparu en 1920 et coexistait avec le ndop de type wukari du Nigéria. Son processus de fabrication passe par deux phases dont la première s'opère au Nord Cameroun et la seconde à l'Ouest, mettant en contact les deux régions.

6 L'obtention d'un tissu ndop original suit un schéma assez étonnant, qui relie le Nord avec le tissage et la teinture à l'Ouest Cameroun où s'effectuent les travaux de surcouture et de finition. La première étape commence au Nord, dans la région de Garoua, où son filage se fait manuellement par des artisans, individuellement ou en groupe de travail d'environ 5 personnes. Puis le tissage s'effectue sur des petits métiers horizontaux par des tisserands. Ces derniers tissent un mince ruban de coton écru, large de $5 \mathrm{~cm}$, appelé " gabaga », qu'ils vendent aux artisans venus de l'Ouest Cameroun (Harter $1986: 144$ ).

7 Une fois rentrés de Garoua, ces artisans cousent les rubans bord à bord pour réaliser des pièces de tissus décorées de larges dimensions. Sur chaque pièce écrue, un maîtreartisan, qui est généralement occupé à d'autres travaux artistiques tels que la vannerie et la poterie, trace, à l'aide d'une fourche de bambou taillé et trempé dans une encre végétale brune, les grandes lignes d'une composition générale et les différents motifs. 
Ils procèdent donc par la méthode de « ligature puis coloration » (Ouédraogo $2011: 7$ ), pendant laquelle des motifs sont créés via des nœuds ou des ligatures du tissu, à l'aide de fils de raphia. Sur une toile de coton blanc, l'artisan dessine les symboles caractéristiques du ndop. Ces symboles sont ensuite cousus au fils de raphia d'un point serré. C'est un travail long et fastidieux qui peut prendre jusqu'à un mois pour compléter la sur-couture du raphia d'une étoffe à motifs très larges de près de $15 \mathrm{~m}$ de long (cf.fig.1). C'est dire que la fréquence de production peut être très courte, même pour un artisan très travailleur.

Fig.1.Travail de sur-couture sur un fond blanc faite par un artisan

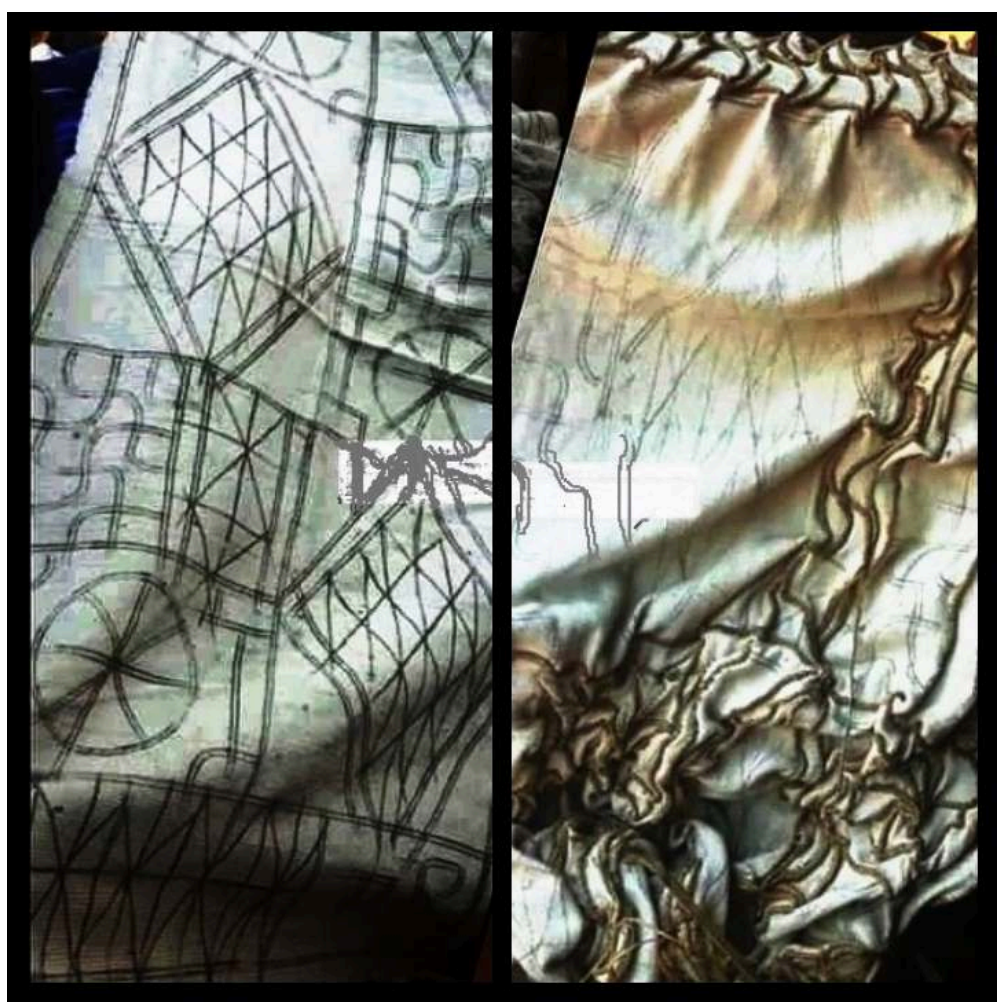

Sur une toile de coton blanc, l'artisan dessine les symboles caractéristiques du ndop

https://www.ndop.me/copie-de-notre-engagement, consulté le 27-03-17

$8 \quad$ Les pièces ainsi brodées retournent à Garoua par voie de route pour être teintes dans l'indigo. La ville de Garoua fut découverte comme centre de teinture en 1893-1894, lors de la visite d'un voyageur allemand, le Dr. Siegfried Passarge ${ }^{3}$ (Lamb 1981 :27). Ici, la teinture se fait en deux étapes. Après avoir baigné pendant environ vingt heures dans une solution de mordant basée sur une lessive alcaline de cendre de bois et d'urine animale, l'étoffe est plongée dans un bain de teinture bleue pour une dizaine d'heures supplémentaires. Elle est ensuite essorée et étendue. L'exposition au soleil est une partie importante du processus car elle entraine l'oxydation de la teinture, donnant ainsi la nuance bleu finale recherchée. La teinture ne doit pas passer à travers les coutures, qui seront enlevées sur le tissu séché (Lamb 1981 :27)

9 De retour à l'ouest, les fils sont décousus par les artisans et les motifs blancs de la décoration ressortent alors sur le bleu indigo du fond. Le fils de raphia joue ici le même rôle que le wax lors du processus d'impression d'étoffes à motifs. Le coloris n'imbibe pas les endroits où se trouvent les coutures en fils de raphia. Ces étoffes, à usages de 
parement avec une forte dose symbolique et fonctionnel, sont principalement destinées aux marchés de Bandjoun, Foumban et Baham, principaux centres de production et de distribution.

\section{Différents usages du ndop}

10 À l'origine, porter le ndop n'est pas un acte anodin. Tenue d'apparat, l'usage du ndop est associé soit au symbolisme du pouvoir et du statut du chef, soit aux coutumes funéraires, agraires et rituelles. L'étoffe sert aussi à fabriquer les costumes de roi, notables et membres des sociétés secrètes. Il sert même de linceul pour envelopper la dépouille du roi ou des notables pour l'enterrement. On décore de coupons de ndop la place des cérémonies funéraires des personnages importants et membres des sociétés secrètes. Les "pleure mort " ont contribué à élargir la clientèle du ndop, car il est également porté par les parents du défunt, et les dignitaires traditionnels bamiléké (Lamb 1981 : 45). À côté de larges bandes utilisées pour la décoration des loges royales, ce tissu sert aussi d'accessoires dans la confection des jupes des danseurs, des boubous, des ceintures ainsi que des bracelets faits en $n$ dop (cf.fig.2).

Fig.2. Jupe de danseur en ndop

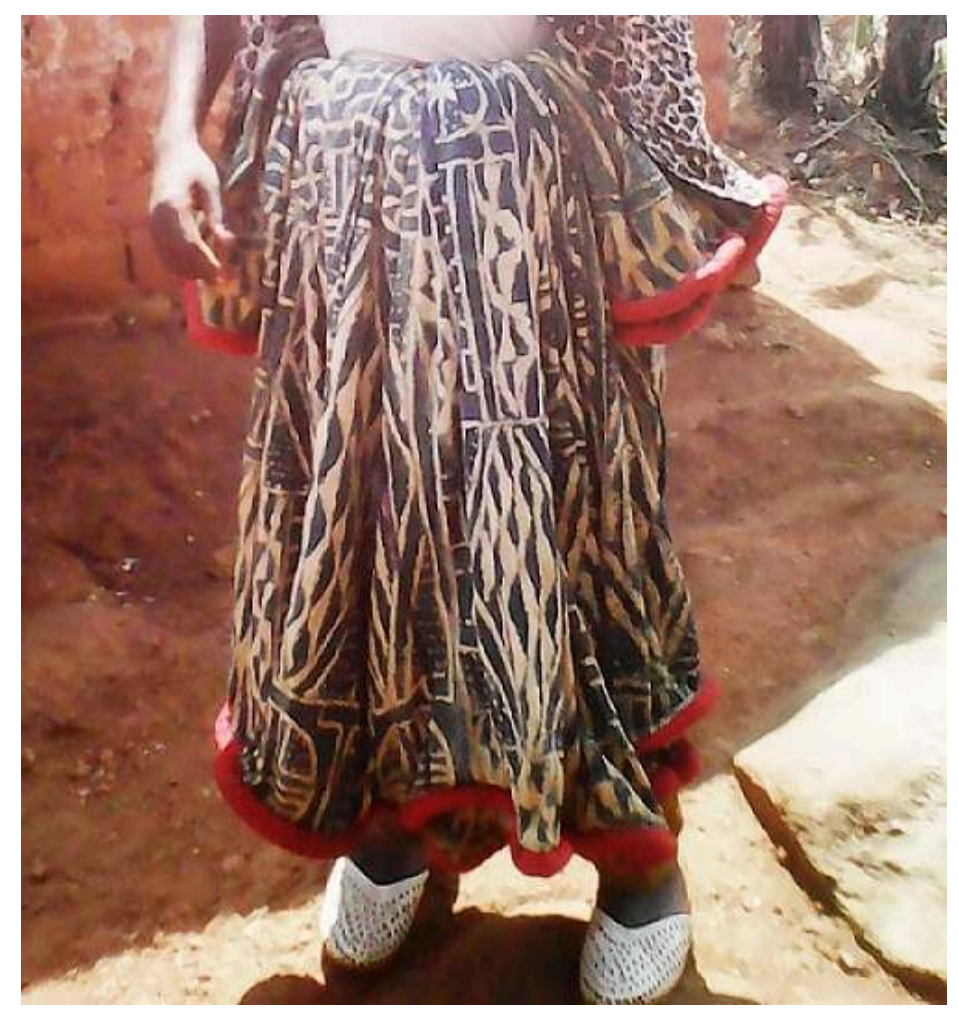

Ce tissu sert aussi d'accessoires dans la confection des jupes des danses patrimoniales et rituelles Cliché Awounang Sonkeng Francine Ulrich, du 21-01-17

Les motifs géométriques figurant sur les tissus ndop sont très représentatifs du rapport de l'homme avec la nature et l'au-delà (la lune, le soleil, les étoiles, les animaux, etc.,). Ils peuvent aussi évoquer l'architecture. Le vocabulaire graphique donne toujours lieu à une interprétation. Il s'agit généralement d'une symbolique abstraite des peaux de léopard, de reptiles, d'animaux venimeux chez les Bamoun et les Bamilékét. De même, 
des motifs géométriques peuvent se rapporter à des scarifications, des ornements d'architecture et de mobiliers (cercles, croix, carrés, triangles, losanges) (Malabon 2016 :77). La croix est une représentation fondamentale : croisement des routes, division de l'espace, expression du " centre du monde » et de la protection qu'il requiert. Leur utilisation sert à transmettre un message de paix et de fécondité. Son graphisme et son iconographie obéissent à des codes, même si on peut observer des variations importantes selon la qualité du support et la dextérité du tisserand qui effectue la sur-couture. Selon Perrois et Notué, le ndop,

« est un véritable livre où l'on peut suivre le message transcrit par le brodeur au moyen de signes. L'artiste est convaincu que l'habileté et le talent dans l'exécution des dessins rendront ces derniers plus efficaces dans leur rôle fonctionnel, au-delà de la valeur marchande du produit. Le ndop a un rapport avec la mort, l'au-delà, le monde invisible. II symbolise le deuil. On décore avec d'amples coupons de ndop la place où se déroulent les cérémonies funéraires des membres des sociétés secrètes. Les parents du défunt, à cette occasion, ont le cou ou les reins drapés d'un morceau de cette étoffe, comme d'ailleurs les statues d'ancêtres royaux. Dans un autre contexte, notamment celui des rites du kè, le bleu est l'expression du surnaturel et des forces de l'esprit. II est aussi le symbole du ciel et de ce fait associé à la pluie. » (Perrois, Notué 1997 : 167).

L'aspect fonctionnel de cette tenue est relevé à l'occasion des deuils, cérémonies funéraires et jadis aussi lors des fêtes agraires. L'usage de vêtements traditionnels à des fins similaires a été observé chez les Ibo du Nigeria, les Ashanti et Akan du Ghana et de Côte-d'Ivoire.

L'obtention de ce vêtement traditionnel authentique au Cameroun se fait à partir de 45000 FCFA pour le demi-boubou (cf.fig.3) et de 90000 FCFA voire plus pour les larges bandes de décoration. Dans une aire dominée par le tissage du raphia, le ndop, étoffe à teintes en réserve, était perçu comme une production ostentatoire réservée aux chefferies (Lamb 1981 :32). Les chefs pouvaient le porter, le donner en récompense à leur sujet ou l'échanger contre des cadeaux avec d'autres chefs ou fons. 
Fig.3. Demi-boubou en tissu ndop

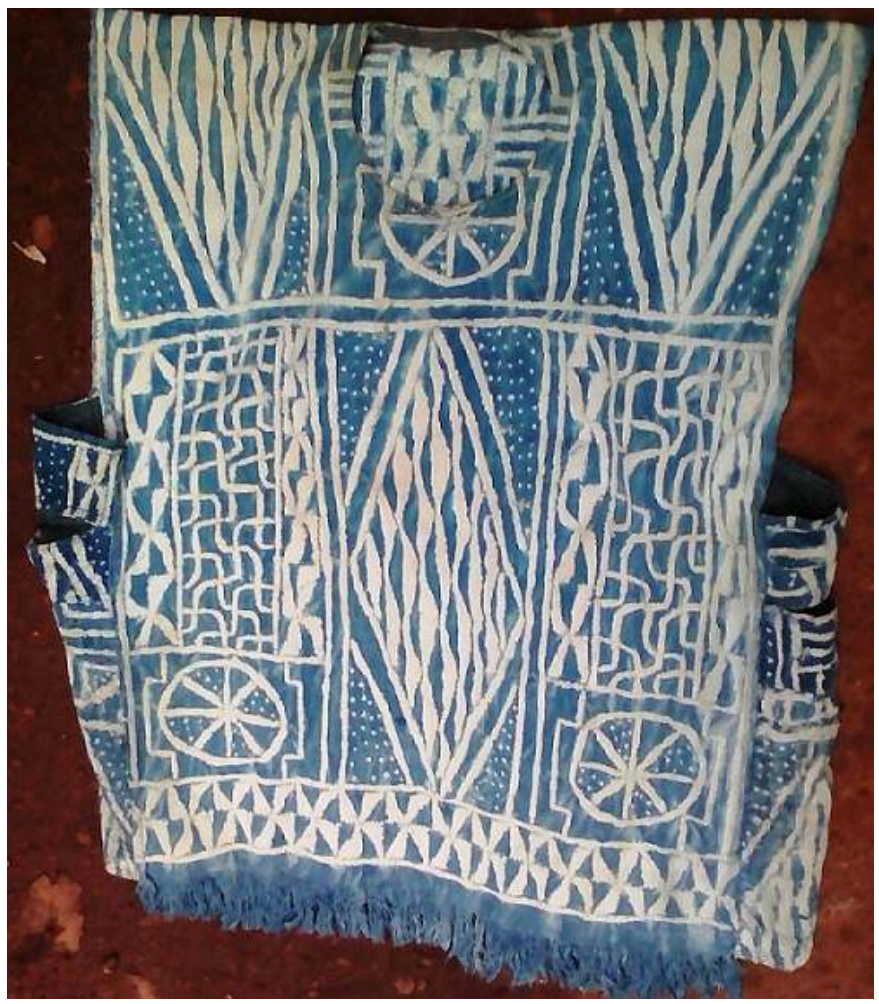

Les motifs géométriques qui se dégagent ici peuvent se rapporter à des scarifications, des ornements d'architecture et de mobiliers (cercles, croix, carrés, triangles, losanges).

Cliché Awounang Sonkeng Francine Ulrich, du 21-01-17

Le procédé de fabrication du ndop laisse cependant de plus en plus la place à de nouvelles formes de productions plus rentables en termes de temps et d'argent, remettant ainsi en cause la fonction symbolique et l'usage même de ce tissu. Cette évolution engendre des conséquences sur sa qualité et son coût.

\section{Évolution et conséquences du processus de fabrication : du ndop traditionnel au ndop industriel}

Les pratiques d'un groupe social sont réglées par les savoirs techniques locaux (Sardan 1996 :128) jusqu'à l'avènement de problèmes inhabituels. Ces savoirs s'insèrent dans l'ensemble des connaissances propres au groupe: conception du monde, sciences, philosophie (Dupré 1991 :94). La fabrication du ndop n’a pas échappé à cette logique.

\section{De l'artisanat à la production industrielle du ndop}

16 L'évolution du processus de fabrication du ndop est observable à plusieurs niveaux, sur le plan de la technique, de l'usage ou même de la valeur marchande du produit.

17 Pour ce qui est de la technique de fabrication du ndop, elle a considérablement évolué avec le temps (Perrois, Notué 1997 :167). La technique de sur-couture au fil de raphia pour les réserves, très longue et fastidieuse, a progressivement laissé place à l'utilisation de la cire, même si le résultat final est moins élaboré. Les motifs sont alors 
créés via une substance imperméable, telle que la cire de bougie ou la pâte à base de manioc (Ouédraogo 2011 :7). Le tissu est ensuite trempé dans des solutions colorantes, généralement fabriquées à partir de teintures industrielles. Ce type de ndop, moins élaboré, est reconnaissable par la légèreté du tissu et son prix bas qui varient entre 7000 et 20000 FCFA. C'est dire qu'elle n'est pas faite d'étroites bandes épaisses, aux fils presque grossiers, cousues bord à bord et décorées de dessins brodés, mais de tissu d'origine industrielle teint de façon artisanale et/ou industrielle.

Un autre type de tissu plus actuel, qui reprend uniquement les motifs du ndop, est fabriqué de façon industrielle avec la technique de production des pagnes Wax à base d'indigo, ou même en impression directe (rotative, digitale, etc.) (CICAM 2006: 9). Aujourd'hui, la CICAM reproduit par exemple des motifs du ndop sur des pagnes appelés «Collection Bantou», en 4 nuances de fond de couleurs : bleu, vert, marron et noir. Cependant, la majorité de ces tissus est d'origine nigériane. Elle ne profite donc pas à l'industrie locale (cf. fig.4).

Fig.4. Tissu reproduisant les motifs du ndop

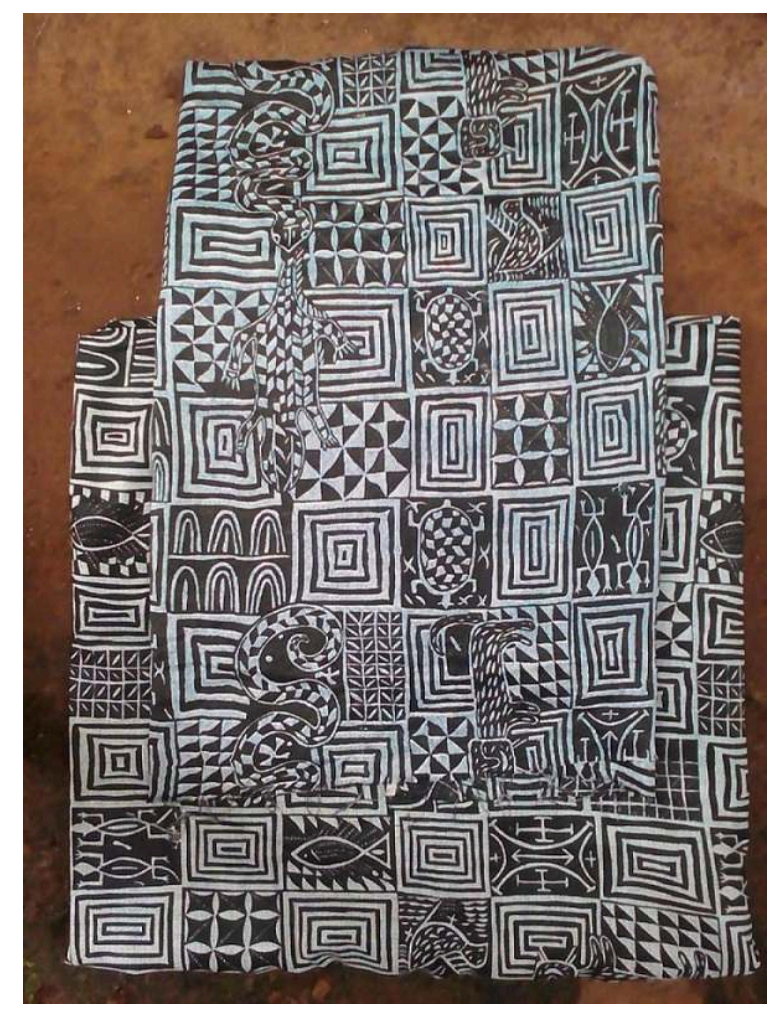

Ce tissu plus actuel qui reprend uniquement les motifs du ndop, est fabriqué de façon industrielle avec la technique de production des pagnes Wax à base d'indigo, ou même en impression directe.

Cliché Awounang Sonkeng Francine Ulrich, du 14-03-2017

19 Les motifs de ce type de tissu sont très réguliers et représentatifs des messages véhiculés par le ndop original à travers le symbole du serpent, de la panthère, du lion, du crocodile, du poisson, du lézard, de la tortue, de l'aigle, etc. Les motifs géométriques blancs et leur arrangement, non teints comme le fond bleu indigo, restent le lieu d'expression des imprimeurs qui, initiés à ces techniques, sont devenus de véritables artistes, mêlant traditions et graphismes esthétiques. Ils produisent généralement du 
tissu de qualité médiocre, qui est vendu aux touristes ou expatriés (Delancey, Mbuh $2010: 59)$.

Toutes ces mutations ont cependant pour seul avantage de permettre à tout un chacun de s'offrir du ndop, même si ce n'est qu'une pale représentation de celui-ci. Comparativement au vrai dont les prix oscillent entre 45000 et 120000 FCFA pour un pays où le salaire minimum est évalué à 36000 francs en 2017, celui-ci ne coûte que le prix d'un pagne normal, soit de 3000 à 6000 FCFA selon son origine et la qualité du support.

21 Ainsi, il existe trois types de ndop : l'originel entièrement l'œuvre des artisans, le semiartisanal constitué de tissu d'origine industrielle, traité par des artisans, mais avec des produits de substitution et l'industriel, pâle copie, dont seuls les motifs et symboles sont inspirés du ndop. Tout ceci n'est pas sans conséquence sur l'usage et la symbolique de cette noble étoffe.

\section{Les implications : du symbole culturel à un vêtement commun?}

Selon Jean Paul Notué, « dans les sociétés ouest camerounaises, comme celle de Baham, les productions plastiques avaient, en premier lieu, une fonction de communication pour les valeurs religieuses, sociales, rituelles et politiques, nécessaires à la cohésion des groupes ». À titre d'exemple, lors des cérémonies d'accueil des visiteurs, les danseurs et musiciens sont revêtus de costumes et/ou de coiffures symboliques, constitués dans différentes étoffes dont la plus importante et la plus prestigieuse est sans aucun doute le ndop (Girault, Galangau-Quérat 2011 :121; Harter 1986 :59). Les chefs suprêmes, garants de la tradition, ont soutenu les activités des artisans en effectuant des commandes et en créant au sein de leur royaume des ateliers de production comme c'est le cas pour la fabrication des tissus ndop au sein du royaume de Baham depuis de nombreuses générations. Conscients de sa symbolique, ils en assuraient ainsi la qualité.

La détérioration de la symbolique du ndop se matérialise dans un musée de la place, lieu censé présenter l'originalité des cultures des peuples du Cameroun. Le tissu décorant un trône Fô au Musée des Civilisations de Dschang est une pâle reproduction industrielle du tissu ndop, pourtant emblème de la civilisation bamiléké. Dès lors, la problématique de la symbolique de cet objet et du rôle des musées est posée. Le tissu exposé présente des motifs imprimés, en porte-à-faux avec les motifs symboliques dessinés sur l'étoffe ndop. Cet objet factice peut-il contribuer à donner aux visiteurs du musée une compréhension de l'importance du ndop dans la culture bamiléké? À l'observation, nous pouvons affirmer à la suite de V. et A. Lamb, que "c'est la production wukari, de moins bonne qualité qui abonde sur les marchés camerounais et les dessins élaborés de haute valeur artistique ne se voient plus que dans les collections des fons, des chefs et de certains musées » (Lamb 1981 :32).

De plus, des habits en ndop sont vendus dans les échoppes touristiques, à Dschang, à Yaoundé et dans certaines boutiques de musées comme à Bamendjinda, alors que la production et la commercialisation étaient autrefois règlementées et contrôlées par les chefs. Le ndop se trouve désormais dans des lieux n'ayant rien de royal (Lamb 1981 :45).

Le ndop participait aussi à des échanges diplomatiques entre chefferies. Pour résoudre certains conflits, les Chefs s'offraient mutuellement ce tissu précieux en signe d'amitié. En effet, si Bandjoun fabrique aujourd'hui des tissus ndop, du temps du Roi Fotso, il 
venait de Foumban et participait à la consolidation des relations entre Bandjoun et Foumban (P.A. 1999 :12).

La vente ou l'achat de ce tissu s'agrégeait à un commerce de prestige réservé à la cour royale. Seul le Chef avait le véritable contrôle sur les transactions liées à l'échange du ndop. Le marché lié à ce tissu est une composante du commerce prestigieux et lucratif des Chefs. Il ne vise pas nécessairement la recherche d'un bénéfice financier. Les Chefs des Hautes Terres de l'Ouest ont participé au commerce des esclaves, de l'ivoire et du tissu ndop dans le seul but d'affirmer leur pouvoir. Raison pour laquelle nous pouvons dire que le commerce des esclaves, de l'ivoire et du ndop est une activité prestigieuse et lucrative. Sa finalité, c'est la distraction, le plaisir, tout en affirmant l'autorité du chef (Malabon 2016 :108)

\section{La crise du ndop : vers un nouveau paradigme}

Si le développement du commerce, de l'artisanat et des productions proto-industrielles comme les tissus ndop, soutient l'économie locale et donne l'occasion à de nouveaux acteurs économiques d'émerger, en retour, il a entraîné un appauvrissement de la fonction symbolique et cultuel de cet objet. En outre, le circuit d'échanges à longue distance qu'il animait a été fragilisé.

\section{Les implications sociales et économiques liées à la production du ndop}

Selon Venice et Alistair Lamb, le processus originel de fabrication du ndop forme un schéma économique assez intéressant pour un système économique traditionnel. En effet, les distances impliquées par le transport des matériaux durant le procédé de fabrication du ndop sont énormes. C'est ce qui amène Barreteau à écrire :

« Il serait difficile de trouver un équivalent du commerce ndop en Afrique. Le plus proche est sûrement le commerce des voiles tukurdi, tissés et teints dans la région de Kano au Nigéria, pour un usage final chez les Touareg et d'autres nomades du Sahara. Il est intéressant d'observer [...] que les étroites bandes utilisées à la fois dans la fabrication nigériane turhdi et la fabrication camerounaise $\mathrm{du}$ ndop sont très similaires et possèdent peut-être des origines communes : le pays hausa » (Barreteau 1997 :236).

En effet, "c'est un trajet total de plus de 2508 kilomètres » (Mwenye 2008 :79), qui implique les tisserands vivant généralement dans les montagnes s'étendant le long de la frontière Cameroun-Nigéria, au Nord de la Bénoué, mais aussi les commerçants Bamiléké et Bamoun qui font des trajets aller-retour pour la sur-couture de raphia à l'Ouest et la teinture au Nord. Ceci induit une coopération économique entre le Nord et l'Ouest Cameroun comme le soulignent V. et A. Lamb : «L'industrie du ndop camerounaise implique une collaboration économique entre les régions du Nord et celle de l'ouest, pour des raisons purement traditionnelles et endogènes, et ne résultent pas de l'incursion d'une domination économique coloniale " (Lamb $1981: 48$ ). Cette longue histoire d'interaction entre les peuples du Nord et ceux de l'Ouest Cameroun animée aussi par le commerce du kola, tend à s'effacer avec la dégradation de l'art traditionnel du ndop. Cette collaboration étroite de deux peuples unis par une pratique 
reste pourtant nécessaire en vue du décollage économique et de la cohésion sociale des groupes.

Ainsi, la fabrication du ndop correspond à un savoir-faire traditionnel à préserver. Il faudrait cependant étudier comment l'adapter en encourageant les brassages de techniques entre les peuples (UNESCO 2004 :8).

\section{Le ndop aujourd'hui : entre désacralisation et démocratisation}

31 L'une des difficultés que subit le ndop aujourd'hui est liée à la mise en avant par la plupart des artisans de sa valeur marchande. En effet, selon Girault et GalangauQuérat:

« En conséquence de leur trop importante mise en scène, qui a pour finalité de défendre la tradition -certains objets du patrimoine culturel d'un peuple, comme à l'Ouest Cameroun-, subissent un effet pervers important; ils deviennent alors des objets commerciaux et perdent leur attrait artistique et surtout leur fonction symbolique. » (Girault, Galangau-Quérat 2011 :114).

Autrefois réservé aux Rois, aux notables et aux sociétés secrètes, le ndop reste un tissu noble et coûteux, mais qui tend à se vulgariser de nos jours. Il fallait auparavant se rendre dans les marchés des grandes chefferies pour y trouver du ndop, ou le recevoir comme cadeau du chef. Aujourd'hui, il est présent sur presque tous les marchés, à des prix accessibles à tous, mais avec, en contrepartie, une qualité médiocre. Si sa fabrication par des maîtres artisans s'est raréfiée, la matière et les motifs apparaissent désormais chez les créateurs de mode et de décoration (Siangne 2004:4). Ainsi, de nombreux créateurs africains se plaisent à le revisiter, à l'instar de l'artiste Ly Dumas ou de Martial Tapolo dont les pièces ont été présentées lors du gala du Gotha Noir en décembre 2012.

Malgré la «démocratisation" de ce vêtement, il est toutefois conseillé de ne pas se vêtir du ndop originel cousu avec des fibres végétales, des écorces d'arbres morts, des cauris, des plumes d'oiseaux, des poils d'animaux, notamment lorsque l'on n'est ni Fô, ni notable (Quartullo 2013 :197). En effet, ce précieux tissu conserve toujours sa valeur cultuelle et culturelle.

\section{Conclusion}

Cette étude a porté sur les transformations dont le ndop, symbole important de la culture bamiléké (Cameroun), a fait l'objet au cours de son histoire. L'objectif consistait à analyser les conséquences de ces mutations sur la qualité d'origine du ndop, sa charge symbolique et les nouvelles caractéristiques de consommations qui en ont découlées. Il est donc à noter que la fabrication du ndop est un héritage qui se transmet de générations en générations ou lors de l'acquisition de la notabilité. Objet précieux issu de l'imaginaire des hommes, le ndop, à travers ses motifs, ses dessins, ses couleurs et croquis, raconte l'histoire des peuples. Il transmet des messages mais a aussi, dans le passé, contribué à créer dans la société des différences sociales entre les hommes de pouvoir et le peuple. À l'origine réservé aux fastes et cérémonies de cour, le développement d'une proto-industrie d'objets culturels met en péril ce tissu qui reste un patrimoine culturel et artistique des peuples Grassfields. 
Si, ailleurs, notamment en Occident, des objets ne sont pas frappés d'interdit, il n'en est pas de même à l'Ouest Cameroun. Il est de notoriété publique que les membres de la communauté bamiléké ne peuvent utiliser le ndop comme accoutrement ordinaire. Sa forte charge symbolique en fait un objet circonstanciel. Ainsi, entre repli identitaire et ouverture, entre démocratisation et conservation du patrimoine, il appartient aux gardiens de la culture de trouver le juste équilibre, pour ne pas brader au nom de la modernité les symboles de nos cultures ancestrales. Aujourd'hui, certains chefs traditionnels s'attèlent à redonner à ce tissu toutes ses lettres de noblesse d'antan, mais le chemin semble encore long avant de pouvoir faire accéder le prestigieux tissu ndop au rang de patrimoine culturel national.

\section{BIBLIOGRAPHIE}

BARRETEAU Daniel, «Les dénominations du coton dans le bassin du Lac Tchad », in BARRETEAU Daniel, DOGNIN René, VON GRAFFENRIED Charlotte (éds.), L'homme et le milieu végétal dans le bassin du lac Tchad. Man and végétation in the Lake Chad basin, Paris, ORSTOM, 1997, p.229-259

BARRETEAU Daniel, DOGNIN René, VON GRAFFENRIED Charlotte (éds.), L'homme et le milieu végétal dans le bassin du lac Tchad. Man and végétation in the Lake Chad basin, Paris, ORSTOM, 1997, p.9-21

CICAM, Monographie de la CICAM, 2006

DELANCEY Mark Dike, MBUH Rebecca Neh, « Ndop cloth », Historical Dictionary of the Republic of Cameroon, Scarecrow Press, 2010

DUPRÉ Georges, Savoirs paysans et développement, Paris, Karthala, ORSTOM, 1991

FOMKONG Nkam Albert, « Sociétés secrètes et notabilités à Baham », dans NOTUÉ Jean-Paul, TRIACA Bianca, Baham. Arts, mémoire et pouvoir dans le royaume de Baham, Milan, Éditions 5 Continents, 2005, p.69-95

GILLOW John, « Ndop resist-dyed cloth of grasslands Cameroon », African Textiles, San Francisco, Chronicle Books, 2003

GIRAULT Yves, GALANGAU-QUÉRAT Fabienne, «Identités culturelles et Patrimoines : Perspectives muséologiques ", dans RODRIGUEZ BARBOSA C., ALVARENGAl L., ABRANTES BARACHO R.M. (Eds), Seminario Internacional Ciencia e Museologia : Universo Imaginario, Universidade Federal do Minas Geraïs, Brasil, 2011, p.7-21

HARTER Pierre, Arts anciens du Cameroun, Arts d'Afrique Noir, Arnouville, 1986

LAMB Venice \& Alastair, Au Cameroun : Weaving-tissage, Douala, ELF-SEREPCA, Cameroun, 1981

LECOQ Raymond, Une civilisation africaine : Les Bamiléké, Paris, Présence africaine, 1953

LEKONFACK NGUENANG, Les fondements du pouvoir dans les chefferies traditionnelles en pays Bamiléké : Le cas de Foto, Mémoire de Maîtrise, Université de Yaoundé I, 1998

MALABON Daris, Patrimoine d'art décoratif et fonctionnel et développement des chefferies Foto et ForékéDschang: 1850-1970, Mémoire de Master, Université de Dschang, 2016 
MOIRA Flanagan, The Royal Cloth of Cameroon, Ph.D. diss., University of Minnesota, 1985

MWENYE AMASSOKO W., Les tissus traditionnels au Cameroun : le tissu ndop et sa place dans l'histoire du Grassland camerounais, Mémoire de Maîtrise, Université de Yaoundé I, 2008

NGUIAFING Jean-Bernard, « Le tissu douop (ndop) à Baham : appropriation d'un art séculaire », dans NOTUE J-P et TRIACA Bianca, Baham. Arts, mémoire et pouvoir dans le royaume de Baham (Cameroun), Milan, Éditions 5 Continents, 2005, p.96-113

NOTUÉ Jean-Paul, La symbolique des arts Bamiléké (ouest-Cameroun) : Une approche historique et anthropologique, Thèse de Doctorat soutenue à l'Université de Paris 1 Panthéon-Sorbonne, 1988

OUÉDRAOGO Carole, « Aux origines des vêtements traditionnels africains », Regard actu, n59, juillet 2011

PERROIS Louis, NOTUÉ Jean-Paul, « Contribution à l'étude des arts plastiques du Cameroun », MUNTU. Revue Scientifique et Culturelle du CICIBA, n²3606, 1987

PERROIS Louis, NOTUÉ Jean-Paul, Rois et sculpteurs de l'Ouest Cameroun : la panthère et la mygale, Paris, Karthala/Orstom, 1997

PERROIS Louis, HARTER Pierre, Les rois sculpteurs : art et pouvoir dans le Grassland camerounais, Paris, Musée National des Arts d'Afrique et d'Océanie, 1993

Politique africaine, (P.A.), $n^{\circ} 76$, La renaissance afro-asiatique, Paris, Kartala, 1999

QUARTULLO Guilliana, L'Art et l'artisanat à Dschang : Du sacré à la profanation (1907-2012), Thèse de Doctorat soutenue à l'Université de Dschang, 2013

SARDAN Olivier, Anthropologie et Développement, Paris, Karthala, 1996

SEIGNOBOS Christian, SCHWENDIMAN Jacques, « Les cotonniers traditionnels du Cameroun », Coton et fibres tropicale, vol.46, $\mathrm{n}^{\circ} 4,1991, \mathrm{p} .309-333$

SIANGNE Paul, Mode et culture, Yaoundé, Lavigne, 2004

SERVET Jean-Michel, « Le principe de réciprocité chez Karl Polanyi, contribution à une définition de l'économie solidaire », Revue Tiers Monde, n¹90, 1986, p.255-273

TARDITS Claude, Les Bamiléké de l'Ouest-Cameroun, Paris, Berger Levrault, 1960

UNESCO, Femmes créatrices : parures et accessoires de mode, Paris, 2004

ZAY Ernest, Histoire monétaire des colonies françaises, Paris, Raymond Serrure, 1904

\section{NOTES}

1. Parmi les utilisateurs camerounais qui appartiennent à de multiples groupes linguistiques, les termes s'y rapportant d'une manière ou d'une autre sont: ndop, doup, ndip, ndob, ndap, ndizindop, ndekong, nzondoup, ndekong, nzondoup, ntieya, ntiesia. Le mot pourrait dériver du ndapbamoum, signalant simplement "coton", ou de la localité des Grasslands, dénommée Ndop, toujours principal centre de tissage, quoique du raphia.

2. Entre 1868 entre 1916, périodes marquées par la présence allemande sur le sol camerounais, la première monnaie officielle fut allemande : le Reichsmark (Zay 1904 :22).

3. Ses écrits sont contenus dans un livre intitulé Adamaoua, qui illustre les fosses de teinture de Garoua.

4. Le serpent à deux têtes : il est le symbole de la sagesse, de la force royale et de la féminité. Il y a aussi une relation biblique à laquelle on peut trouver deux sens, celui du tentateur, mais aussi 
celui du salut. L'araignée : elle a le don de lire l'avenir ; elle exprime aussi la joie de vivre et la sagesse. Le lézard: une légende dit qu'au commencement Dieu envoya deux messagers sur la terre : le caméléon qui devait annoncer aux hommes la résurrection après la mort et le lézard qui devait annoncer la mort sans retour, le lézard arriva le premier. La spirale : elle représente la solidarité. Le dos de tortue représente la sagesse, l'adresse et la prudence. Les Cauris représentent la richesse et la fécondité : chaque reine porte à chaque événement, soit des colliers de cauris, soit les coquillages insérés dans les tresses. Le fer de lance est le symbole de la force royale.

\section{RÉSUMÉS}

Étoffe la plus emblématique du pays Grassfields, le ndop, riche en symbole et en histoire, fait partie de la culture bamiléké. Très utilisé dans les sociétés secrètes et lors cérémonies rituelles, ce tissu reste l'un des symboles de la structure sociale bamiléké et bamoum. Il est le reflet d'une tradition textile perpétuée depuis des générations. Cependant, sa fabrication et son usage ont subi une certaine libéralisation, altérant ainsi l'essence même d'un des symboles culturels d'un peuple. Quelles sont les mutations survenues dans la chaine opératoire de fabrication du tissu ndop et comment ont-elles impacté sur sa perception et son usage? L'analyse de différentes sources permet d'affirmer que le ndop, tissu originaire du Nord-Cameroun, était tissée avec des matériaux traditionnels. Ce tissu est porteur d'une charge historique, sociale et culturelle qu'il importe de valoriser. Cependant la production industrielle a fortement détérioré sa qualité et sa symbolique.

Rich in symbols and history and part of the Bamileke culture, the Ndop fabric is the most emblematic fabric of the Grassfields country. It carries a historical, social and cultural charge which it is important to enhance. This fabric originating in North Cameroon and woven with traditional materials reflects a textile tradition perpetuated for generations, and remains one of the symbols of the social structure Bamileke and Bamoun. Most of them are used in secret societies and ritual ceremonies. However, their manufacture and use have been subject to some liberalization. Their industrial production has seriously deteriorated their quality and symbolism. This article analyzes the mutations that have occurred in the Ndop tissue production chain and how it has affected their perception and use.

\section{INDEX}

Mots-clés : histoire des techniques, textile, savoir local, savoir traditionnel, culture matérielle Keywords : history of technology, textile, traditional knowledge, local knowledge, material culture

Thèmes : Un objet une technique 


\section{AUTEURS}

\section{FRANCINE ULRICH AWOUNANG SONKENG}

Francine Ulrich Awounang Sonkeng est doctorante en économie et histoire sociale, à l'Université de Dschang (Cameroun). Ses recherches sont axées principalement sur l'histoire de l'industrie et des entreprises, l'histoire des techniques et de l'artisanat, le textile, l'industrie de la mode et de l'habillement. Elle travaille actuellement sur les dynamiques du secteur textile et de l'habillement au Cameroun entre 1919 et 2010.

\section{JULES KOUOSSEU}

Jules Kouosseu est maître de conférences à l'Université de Dschang (Cameroun). Spécialisé en Histoire économique, sociale et culturelle, il a publié récemment : « Le patrimoine culturel des Bamoun et des Banso du Cameroun à l'épreuve de la frontière franco-britannique (1916-1961), in FOUELLEFAK K. Célestine et NZESSE Ladislas (dir.), Patrimoine culturel africain : matériau pour l'histoire, outil de développement, Paris, L'Harmattan, 2017, p.263-281. Il étudie actuellement les relations entre la colonisation et les mutations dans l'art de bâtir chez les Bamilékés du Cameroun. 